\title{
Single-stage deformity correction in children with neurogenic kyphoscoliosis: nuances and a proposal for risk stratification
}

\author{
Suhas Udayakumaran, MCh, ${ }^{1}$ Sajesh K. Menon, $\mathrm{MCh},{ }^{2}$ Chiazor U. Onyia, MBBS, ${ }^{3}$ and \\ Naveen Tahasildar, MS ${ }^{4}$
}

Divisions of ${ }^{1}$ Paediatric Neurosurgery and ${ }^{2}$ Spine Surgery, Department of Neurosurgery, Amrita Institute of Medical Sciences and

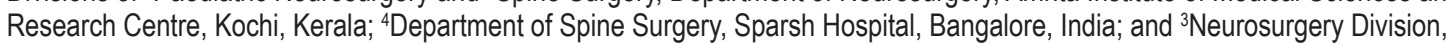
Department of Surgery, Obafemi Awolowo University Teaching Hospitals Complex, lle-Ife, Nigeria

OBJECTIVE Neurogenic kyphoscoliosis in pediatric patients has varied causes and diverse management options. The most common management strategy is to stage the orthopedic and neurosurgical aspects of the correction at an interval of 3-6 months from each other. The aim of this study was to report the authors' experience with correction of deformity in children with associated neurological abnormalities requiring intervention as a single-stage surgical treatment.

METHODS Of 591 pediatric patients with scoliosis treated at the Amrita Institute of Medical Sciences and Research Centre between January 2001 and January 2011, the authors identified all patients with neurogenic kyphoscoliosis who underwent deformity correction and a neurosurgical procedure in the same surgical session when they were younger than 15 years. Data regarding the demographic details, preoperative Cobb angle/kyphotic angle, surgical details, postoperative complications, cost of the procedure, and long-term neurological outcome were collected for each case and analyzed. Based on the results of this analysis, the authors propose a 2-category risk stratification system for the timing of deformity correction depending on the primary neurogenic etiology.

RESULTS Ten cases involving patients with neurogenic kyphoscoliosis requiring both deformity correction and neurosurgical procedure met the criteria and were included in the analysis. All 10 patients were younger than 15 years; their mean age was 10.8 years. The average Cobb angle was $59.5^{\circ}$. Five patients underwent foramen magnum decompression, 5 patients underwent spinal cord detethering, and 1 patient had intraspinal intradural tumor excision. The mean estimated blood loss was $1177 \mathrm{ml}$ (range 700-1550 ml), and the mean operative time was 4.4 hours (range 3-5.5 hours). There were no intraoperative complications. None of the patients had any new postoperative neurological deficits. The mean postoperative Cobb angle of the main thoracic curve was $12.6^{\circ}$.

CONCLUSIONS Simultaneous deformity correction along with a definitive neurosurgical procedure in patients with neurogenic kyphoscoliosis can be performed without added morbidity and avoids both the added costs and the risk of complications associated with a second surgery. The risk stratification system proposed in this paper may serve as a guideline for case selection and help to optimize outcome.

https://thejns.org/doi/abs/10.3171/2017.7.FOCUS17348

KEY WORDS scoliosis; neurogenic kyphoscoliosis; complex spinal deformities; tethered cord; Chiari malformation

$\mathrm{N}$ EUROGENIC kyphoscoliosis in children has varied etiology with similarly diverse management options. ${ }^{6,14}$ Association of neural axis abnormalities as well as intraspinal abnormalities with various types of kyphoscoliosis is well documented. ${ }^{9}$ These cases are commonly characterized by an early onset and a double curve. ${ }^{9}$ Commonly documented neurogenic pathologies that may lead to spinal deformity include Chiari malformation
Type I (CM-I) (particularly with syringomyelia), tethered cord syndrome, and intramedullary as well as intradural extramedullary spinal tumors. ${ }^{3} \mathrm{CM}-\mathrm{I}$ with associated syringomyelia is the most common of these abnormalities, followed in order by tethered cord (diastematomyelia [also termed split cord malformation] or dysplastic filum terminale) and intramedullary tumors. ${ }^{3,13}$

Kyphoscoliosis associated with neurological abnormal-

ABBREVIATIONS AP = anteroposterior; $\mathrm{CM}=$ Chiari malformation; $\mathrm{CM}-\mathrm{I}=\mathrm{CM}$ Type I.

SUBMITTED June 1, 2017. ACCEPTED July 10, 2017.

INCLUDE WHEN CITING DOI: 10.3171/2017.7.FOCUS17348. 
ities always poses a special challenge to the treating physicians, and a systematic approach is required for handling both problems. ${ }^{4,5,11}$ Neural axis abnormalities have been described as occurring in approximately $20 \%$ of patients with congenital kyphoscoliosis. ${ }^{13}$ On the other hand, the reported incidence of intraspinal abnormalities in juvenile and infantile idiopathic scoliosis varies widely from $23 \%$ to $88 \% .{ }^{9,13}$

The optimal timing of surgical correction of deformity with respect to the treatment of the associated neurological abnormality has, however, remained a subject of debate. ${ }^{8,9}$ The most practiced line of management, as reported in the literature, is to stage the orthopedic and neurosurgical procedures at an interval of 3-6 months apart. ${ }^{7}$ Some authors have previously recommended a staged approach (with the interval between stages ranging from 6 weeks to 6 months) in which the underlying neurological cause is treated first, followed by correction of the kyphoscoliosis..$^{1-3,12}$ Staged operations are said to result in less morbidity in high-risk patients. ${ }^{5}$

In this retrospective study, we review our experience with single-stage, concurrent orthopedic and neurosurgical management of kyphoscoliosis in children with associated neurological abnormalities at our institution and assess the safety and effectiveness of this approach. We suggest a guideline for the risk stratification of candidates for such an approach with the goal of helping to optimize outcomes.

\section{Methods}

This study was a retrospective analysis of all cases involving patients younger than 15 years of age who underwent both deformity correction and a neurosurgical procedure to treat the underlying cause during the same surgery at Amrita Institute of Medical Sciences and Research Centre between January 2001 and January 2011. Only patients who had simultaneous procedures for both indications were included in the study. Data regarding the demographic details, preoperative Cobb angle/kyphotic angle, surgical details, postoperative complications, and cost of the procedure as well as long-term neurological outcomes for each patient were collected from the hospital information system. Radiological parameters were analyzed using Amrita MedVision software (Amrita Technologies). Urodynamic studies were routinely performed as part of the preoperative diagnostic and baseline evaluation and during follow-up in all cases.

\section{Results}

A total of 591 pediatric patients underwent scoliosis surgery at our institution during the study period. Of these patients, 10 (5 male and 5 female) met the criteria for inclusion in the study. The mean patient age at the time of surgery was 10.8 years. Table 1 shows patient demographic and clinical characteristics as well as a summary of the neurosurgical and orthopedic procedures performed.

The patients' mean preoperative Cobb angle was $59.5^{\circ}$, and their mean preoperative flexibility was $36.5 \%$. Of the 10 patients, 7 had single thoracic curves, 2 had double thoracic curves, and 1 had a thoracolumbar curve. All of the thoracic curves were left sided. The mean apical vertebral translation of the main thoracic curve was $4.21 \pm 1.32 \mathrm{~mm}$. Five patients underwent foramen magnum decompression, and 2 of these 5 patients underwent syringosubarachnoid shunt placement for holocord syringomyelia (1 patient had syringomyelia, which was not addressed directly, and 2 patients had isolated Chiari malformation $[\mathrm{CM}]$ without syringomyelia). Five patients had detethering, and 1 patient had intraspinal intradural tumor excision. Of the 5 patients who had detethering, 2 had undergone myelomeningocele repair in infancy; both of these patients had a deformity with progressive neurological deficit, and in both cases detethering was performed along with deformity correction. Of the 3 patients with diastematomyelia (see Case 5 patient shown in Figs. 1 and 2), 1 had syringomyelia and 2 had no symptoms but had progressive scoliosis. Therefore, only 2 patients with tethered cord had no symptoms other than scoliosis.

Whole-spine standing anteroposterior (AP) and lateral radiographs with supine side-bending views were obtained to assess the severity of deformity in each case and determine which levels to fuse. All radiographs were saved as DICOM images on the hospital information system (Amrita MedVision software, Amrita Technologies) and various radiographic measurements were calculated using built-in digital tools. Clinical photographs were taken from the back (standing, sitting, and bending forward) to assess the cosmetic parameters, including truncal balance, shoulder balance, and rib hump. Whole-spine MRI, including the craniocervical junction, was performed to assess neurological abnormalities. CT scans of the whole spine were performed to assess for bony bar in split cord malformations and also to assess the bony morphology in congenital scoliosis.

The orthopedic and neurosurgical procedures were performed sequentially in a single surgical setting. The typical protocol followed was to perform the neurosurgical procedure as the first step and then proceed with deformity correction. Neuromonitoring was used for most of the cases, with assessment of both somatosensory evoked potentials and motor evoked potentials throughout the surgery. A neurophysiologist was available in the operating room to monitor the potentials, because only a manual neuromonitoring system was available. Convex hemiepiphysiodesis was performed in 3 patients, and posterior scoliosis correction was performed in 7 patients. Foramen magnum decompression was performed in 5 patients and detethering of the cord was performed in 5 patients (including 2 patients previously operated on for myelomeningocele in infancy; Figs. 3-6), and 1 patient underwent intradural tumor (neurofibroma) excision. The mean estimated blood loss was $1177 \mathrm{ml}$ (range 700-1550 ml), and the mean operative time was 4.4 hours (range $3-5.5$ hours). There were no intraoperative complications. None of the patients had any new neurological deficit or showed any evidence of deterioration from their preoperative neurological status, and there were no intraoperative neuromonitoring alerts. There were no significant postoperative complications. There were no CSF leaks. The mean postoperative Cobb angle of the main thoracic curve was $12.6^{\circ}$ and the average deformity correction was $78.2 \%$ (range $62.1 \%-95.2 \%$ ). However, 1 patient developed adding-on phenomenon, 


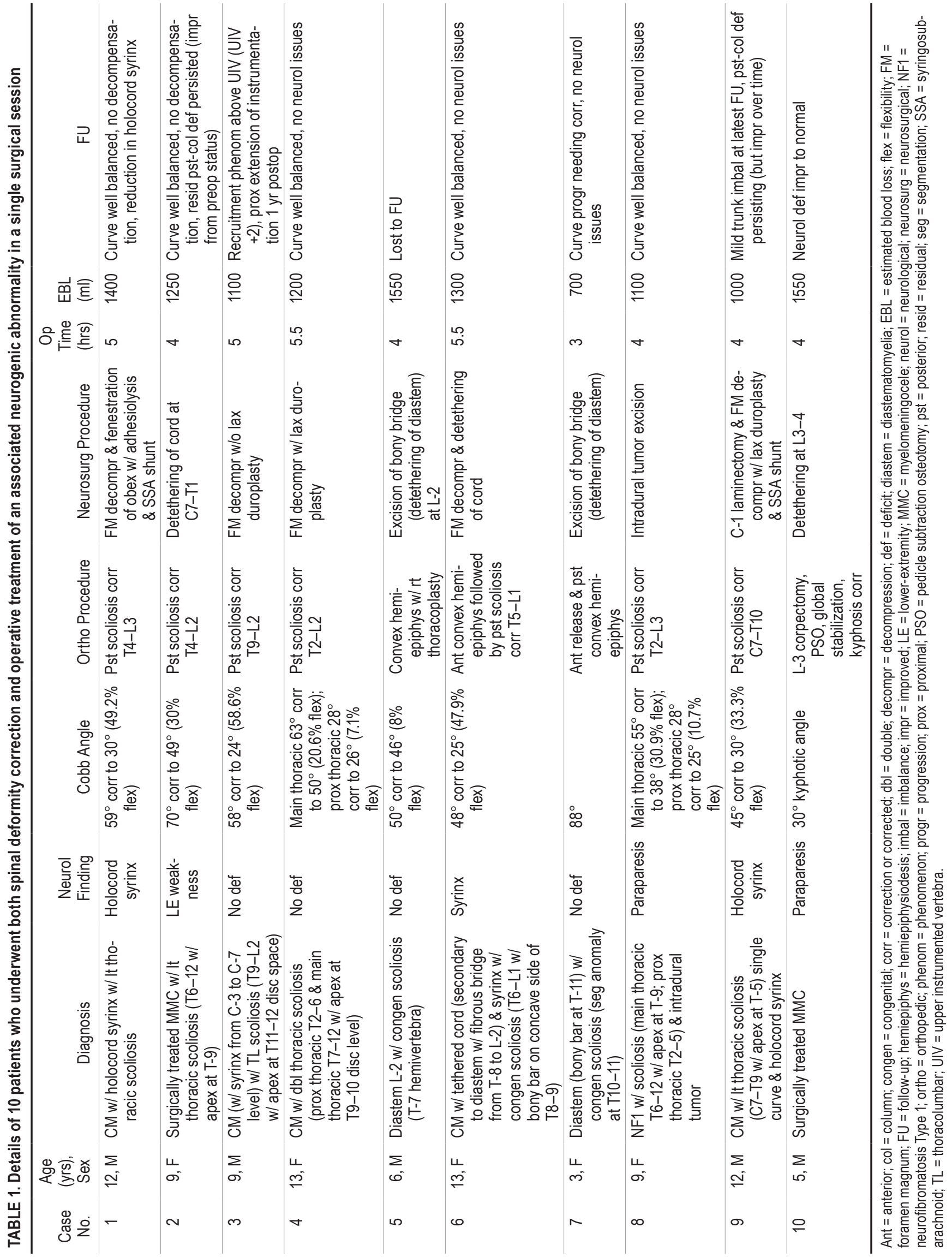



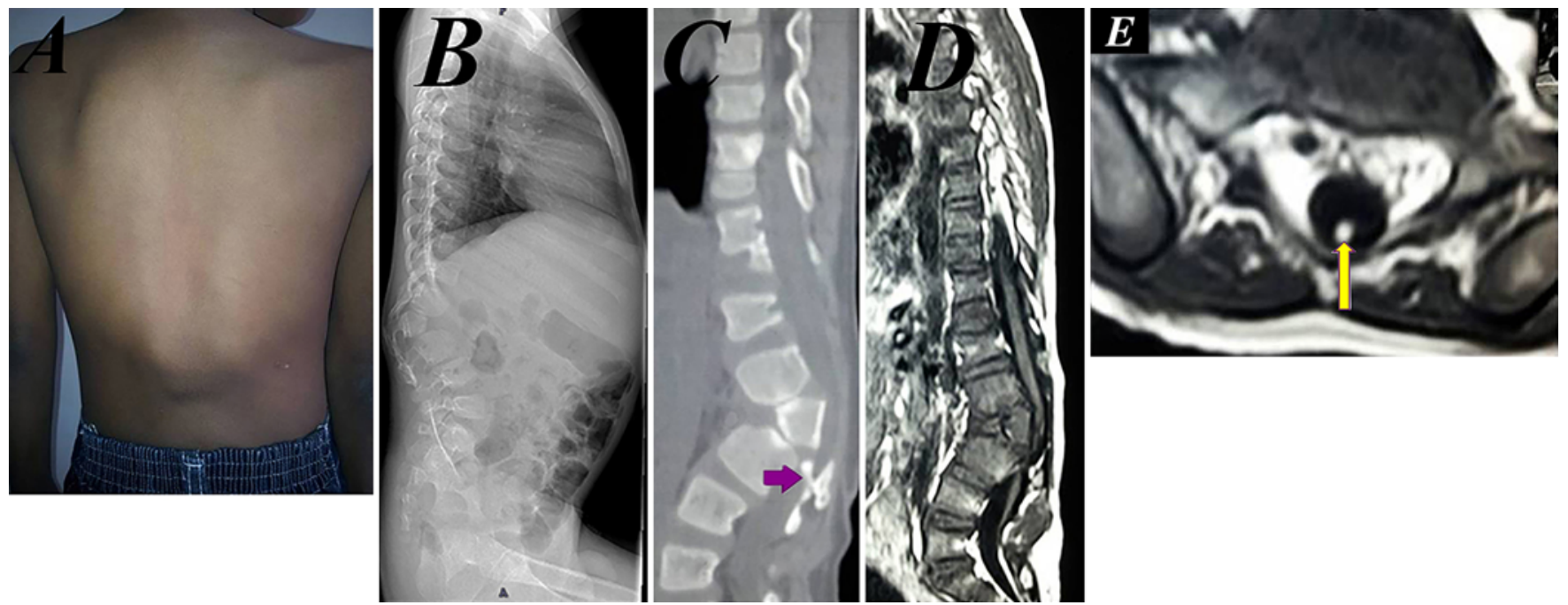

FIG. 1. Case 5. Preoperative photograph of and radiological images obtained in a 6-year-old boy with congenital scoliosis, a T-7 hemivertebra, diastematomyelia, and a lipomatous filum terminale. A and B: Photograph and lateral radiograph. C: Sagittal CT reconstruction showing the split cord (arrow). D and E: Sagittal (D) and axial (E) T1-weighted MR images showing the lipomatous filum terminale (arrow in E).

with proximal progression of the main thoracic curve, during the follow-up period and required proximal extension of instrumentation for correction.

\section{Discussion}

Neurogenic kyphoscoliosis has been seen in a variety of neurological disorders, including CM, syringomyelia, spinal dysraphism with associated tethering of the cord, and spinal cord tumors. ${ }^{2,3,6,14}$ It is important to recognize the underlying neurological factors involved in the kyphoscoliosis, because correction of these factors may stabilize or even reverse the deformity in some cases ${ }^{2,14}$ and is essential to avoid neurological morbidity. For this reason, a whole-spine MRI is warranted not only in patients with congenital scoliosis but also in those with idiopathic scoliosis. ${ }^{4,6,11}$ It has been reported that up to $42 \%$ of patients with $\mathrm{CM}$ have scoliosis and approximately $50 \%-75 \%$ of patients with CM have syringomyelia. ${ }^{3}$ Suboccipital decompression in patients with CM has been found to improve or halt the progression of scoliosis. ${ }^{3}$

Even though syringomyelia is well known to be associated with scoliosis, the underlying cause of the connection between syringomyelia and scoliosis remains unclear. ${ }^{16}$ It has been demonstrated that patients with both CM-I and syringomyelia who have foramen magnum decompression surgery before they reach 12 years of age have a high probability of spontaneous improvement of scoliosis following complete or partial resolution of the syrinx, while scoliosis will tend to progress over time instead of regressing if the decompression is performed after 12 years of age..$^{16}$

About $40 \%$ of patients with split cord malformation will eventually develop scoliosis. ${ }^{6,11} \mathrm{~A}$ faster progression of scoliosis (estimated at $>10^{\circ}-12 \%$ year) is commonly observed with tethered cord. ${ }^{8}$ Adequate detethering has been shown to improve or stabilize the scoliosis., ${ }^{2,3}$ On the other hand, cord detethering is also mandatory prior to deformity correction to prevent neurological injury. Retethering following surgery is frequently seen in patients with split cord malformations, myelomeningocele, and transitional lipomas but rarely occurs in lipomatous filum terminale. ${ }^{9}$ Retethering is rarely observed earlier than 6-12 months after surgery, which is the basis for staging the orthopedic and neurological procedures around 4-6 months after detethering. $3,8,9$

Interestingly, adding to the debate, a contradictory
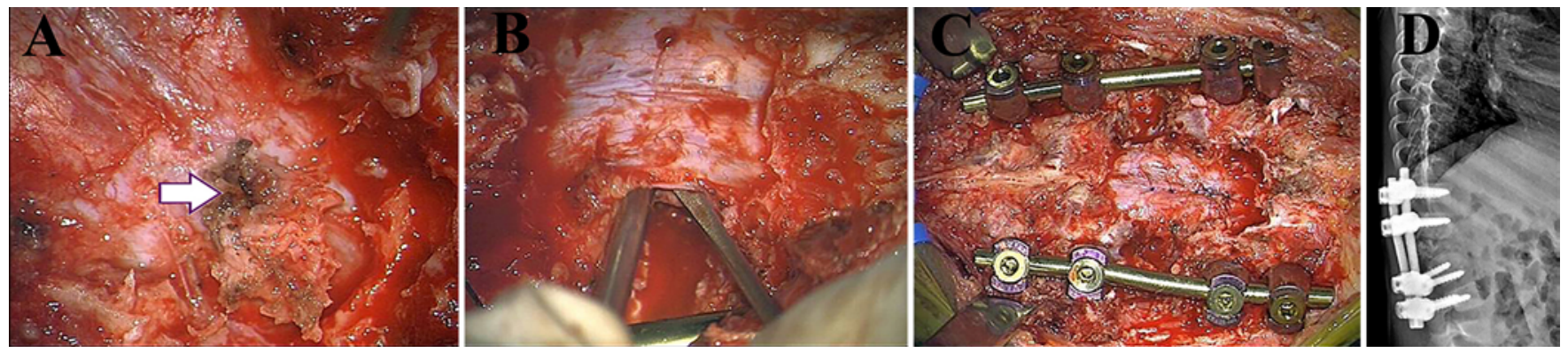

FIG. 2. Case 5. Intraoperative photographs and postoperative radiograph obtained after posterior vertebrectomy and excision of distematomyelia, sectioning of lipomatous filum, and stabilization. A: Photograph of the split cord (arrow). B: Photograph obtained after vertebrectomy. C: Photograph obtained after placement of instrumentation. Note the sutured dural tubes. D: Lateral radiograph obtained after completion of surgery demonstrating accurate placement of instrumentation. 

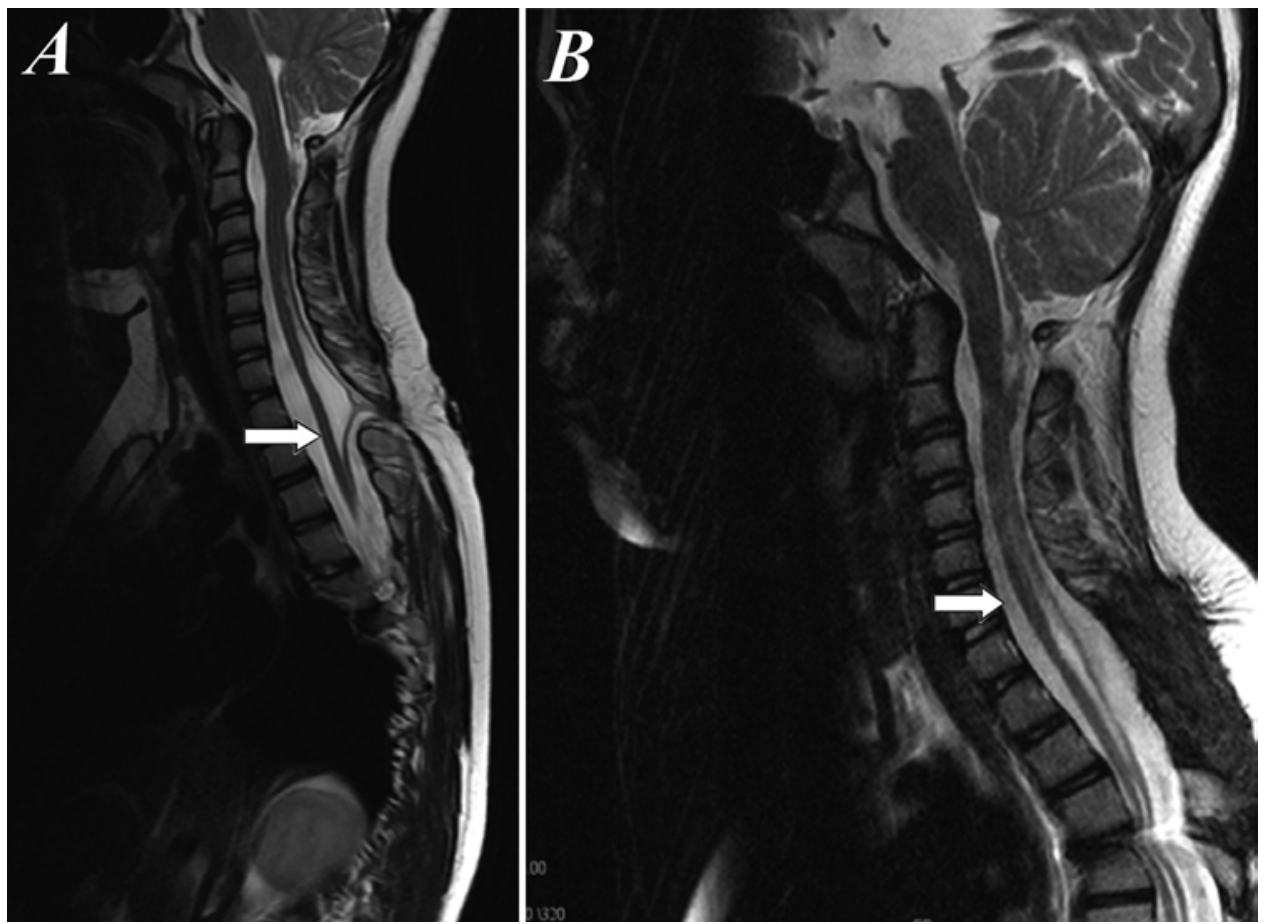

FIG. 3. Case 2. Sagittal T2-weighted MR images obtained in a 9-year-old girl with left thoracic scoliosis (T6-12 with apex at T-9) and a previously treated myelomeningocele (operated on in infancy). A: Preoperative MR image showing dorsal tethering (arrow). B: Postoperative MR image demonstrating successful release of the tethered cord.

opinion was expressed in the report of a recent study by Shen et al., ${ }^{15}$ who questioned the role of detethering in split cord malformation. Their evaluation of a treatment strategy for congenital scoliosis associated with split cord malformation in 214 patients who had either Type I or Type II split cord malformation (according to the classification by Dachling Pang) interestingly revealed that congenital scoliosis patients with split cord malformation who had no neurological deficits could safely undergo correction of the spinal deformity without prophylactic detethering; although there were several cases of transient postoperative neurological deficits, all resolved fully, and at a mean follow-up period of 37 months, no patient had any new permanent neurological deficit. ${ }^{15}$

The surgical strategy in patients with neurogenic kyphoscoliosis has long been a matter of debate. ${ }^{8,9}$ Many studies recommend a staged approach for performing spinal cord detethering as well as management of any syrinx present before correction of the deformity. ${ }^{5}$ Only a few studies have investigated carrying out single-session surgery for the treatment of both neurogenic and orthopedic problems in these patients. ${ }^{8-10,13}$ However, the objective evidence to support the staged approach is lacking. ${ }^{9}$ Recent studies have questioned the necessity of staging and suggested an alternative approach of single-session surgery. ${ }^{8,10}$ While comparing concurrent versus staged procedures, Mehta et al. ${ }^{8}$ found no wound complications or any new onset of neurological deficits in the concurrent-surgery group. They also found that patients in the staged-surgery group had greater blood loss, longer operative time, more wound complications, and longer length of hospital stay. A second surgical procedure in a staged approach may entail an increased risk of wound complications due to a poorly healed surgical field and/or poor nutritional status of the patient. ${ }^{8-10}$ Apart from avoiding these complications, the concurrent-surgery strategy also reduces the total cost of treatment. ${ }^{8,10}$ Additionally, studies have found that patients return to full functional status much more rapidly after a concurrent approach. ${ }^{8,10,13}$ However, it has been suggested that only selected patients are likely to benefit from such a combined approach. ${ }^{9}$

It should be mentioned that Wang et al. ${ }^{16}$ reported single-stage correction of moderate to severe scoliosis in adolescents with CM-I using posterior vertebral column resection. According to these investigators, posterior ver-
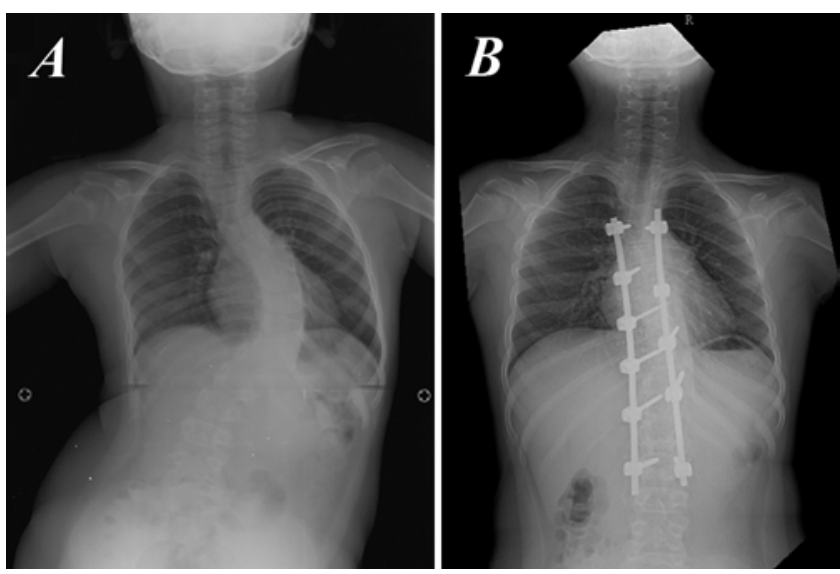

FIG. 4. Case 2. AP radiographs obtained before $(\mathbf{A})$ and after $(\mathbf{B})$ placement of instrumentation. 

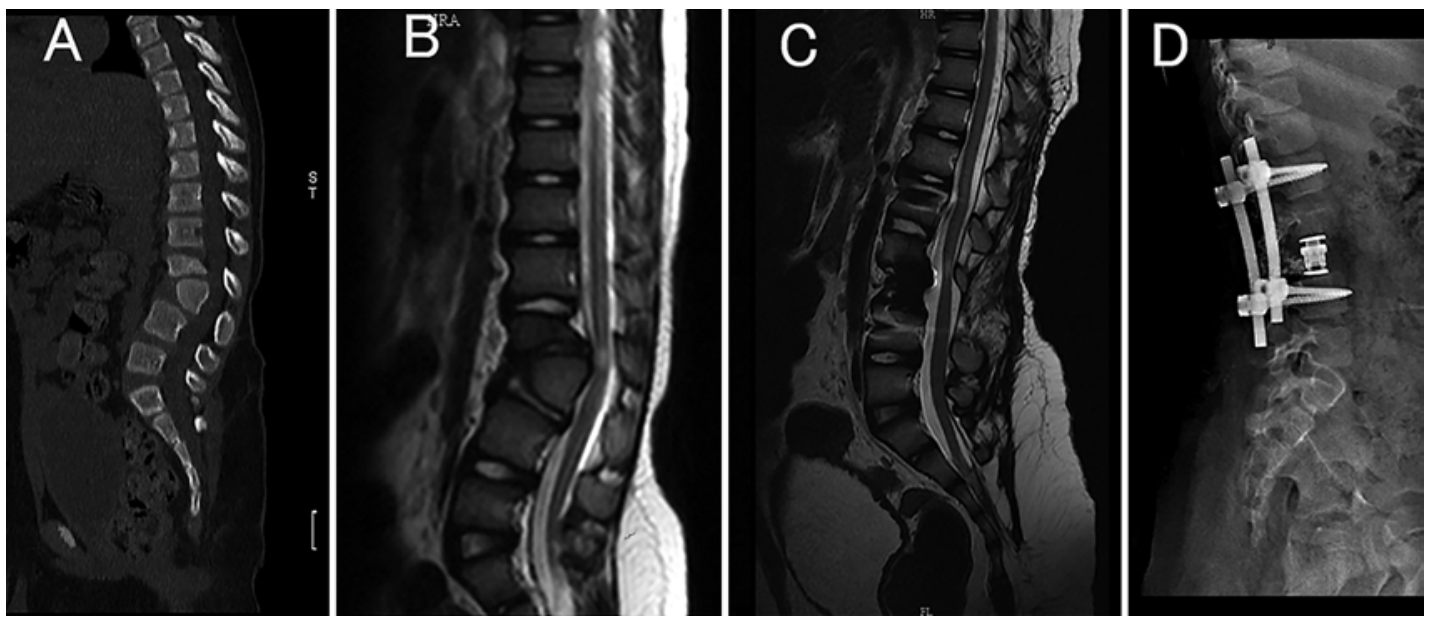

FIG. 5. Case 10. Radiological images obtained in a 5-year-old boy who presented with progressive deficit and kyphosis after having been operated on for myelomeningocele in infancy and underwent L-3 corpectomy and global stabilization along with detethering. A: Sagittal CT reconstruction showing an L-3 hemivertebra. B: Sagittal T2-weighted MR image showing L-3 kyphosis and cord changes. C: Postoperative sagittal T2-weighted MR image showing improvement of the cord changes and lordotic spine. D: Postoperative lateral radiograph showing global stabilization.

tebral column resection can effectively shorten the spine and reduce the tension on the spinal cord, without suboccipital decompression. Wang et al. did not report any iatrogenic neurological deterioration, and even more importantly, they observed a gradual reduction in syrinx size following posterior vertebral column resection procedures. ${ }^{16}$

\section{Nuances, Risk Stratification, and Limitations}

\section{Risk Stratification to Guide Decision Making}

On the basis of our experience, for the purpose of optimizing safe management, we recommend stratification of the risk of simultaneous correction of spinal deformities and associated neurological abnormalities in patients with neurogenic kyphoscoliosis into 2 categories as follows.

- Category 1-No Additional Risk. This category includes patients with CM without a syrinx, those undergoing detethering for which 2 separate incisions are involved (e.g., patients with a lipomatous filum terminale), and those with intraspinal tumors. For patients in this category, there may be a benefit to staging apart from logistical reasons and convenience for the surgical team. We believe that, apart from the logistics of simultaneous surgery, procedures such as foramen magnum decompression, tethered cord release, and repeat
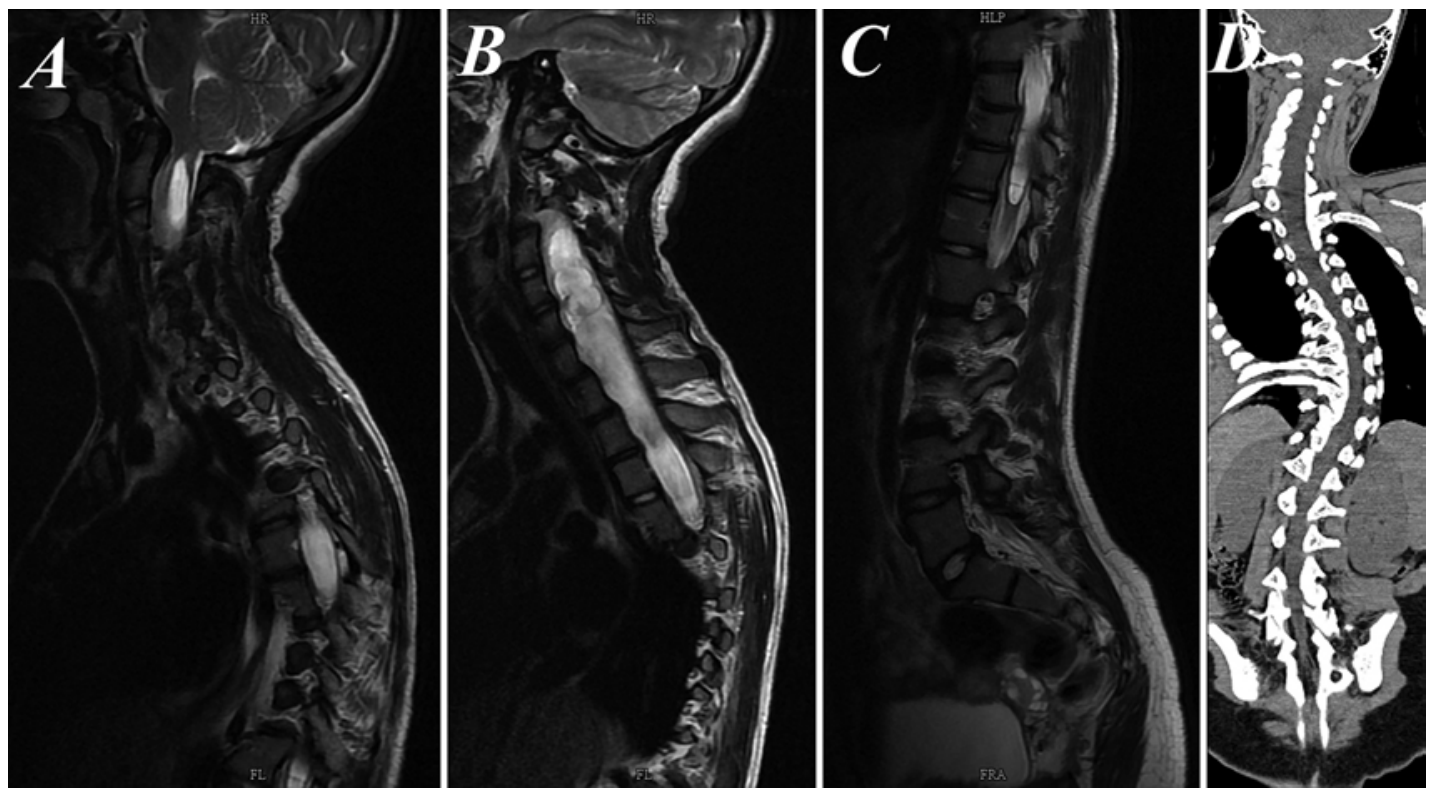

FIG. 6. Case 9. Preoperative sagittal T2-weighted MR images (A-C) and coronal CT reconstruction (D) obtained in a 12-year-old girl with $\mathrm{CM}$, holocord syringomyelia, and left thoracic scoliosis. Note the extensive cervicothoracic syrinx seen in the preoperative images and compare to the almost compete resolution shown in the postoperative images in Fig. 7. 

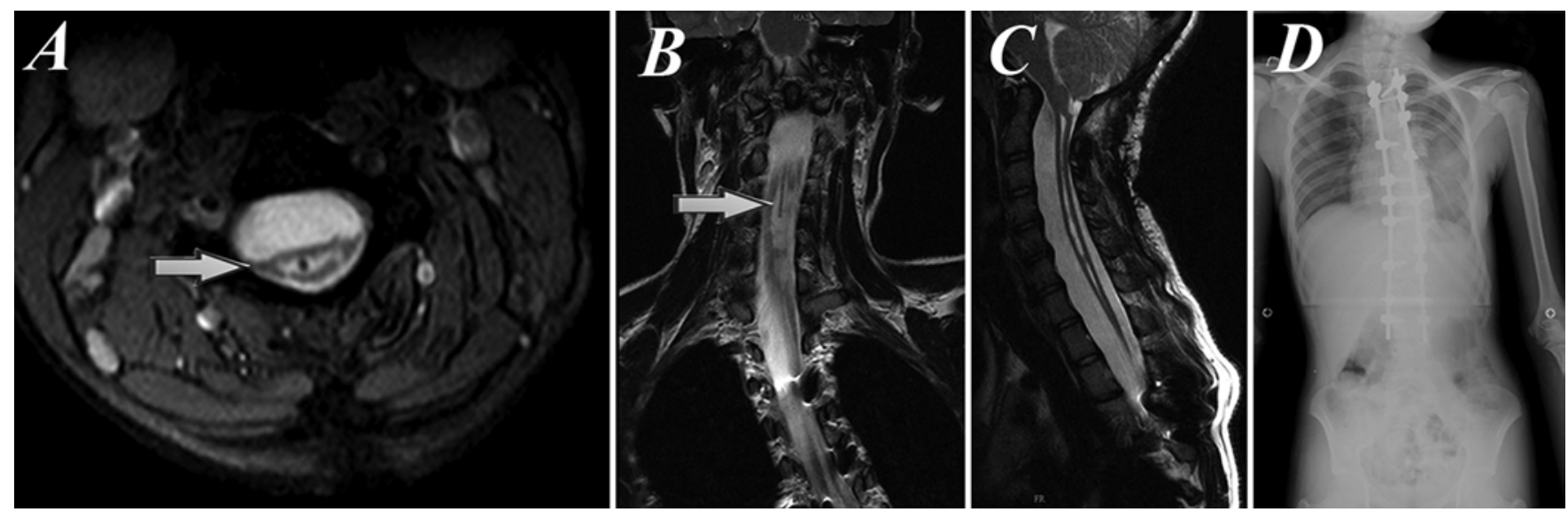

FIG. 7. Case 9. Postoperative axial (A), coronal (B), and sagittal (C) T2-weighted MR images and AP radiograph (D) obtained 6 months after revision of foramen magnum decompression with adhesiolysis and placement of a syringosubarachnoid shunt (arrows). Note the resolution of the syrinx in the MR images.

detethering in patients who have previously undergone surgical treatment of myelomeningocele can all safely be performed in the same sitting for 2 reasons: 1) the cord is not compromised, and 2) the incisions are separate.

- Category 2-Higher Risk. In the presence of significant syringomyelia, there is an increased risk that the cord is already compromised. If patients require surgery for a tethered cord as well as deformity correction, especially when both surgical procedures require the same incision, a putative higher risk of meningitis and CSF leak may be anticipated.

Staging of the neurosurgical and orthopedic procedures has its merits and is definitely a valid option. In cases of deformity associated with compromised neurological function, the surgical procedure of deformity correction may theoretically be damaging to the cord. Our experience has shown, however, that a safe surgery and an optimal outcome are possible even in these situations.

In patients with holocord or significant syringomyelia, we performed and would advocate revision foramen magnum decompression and placement of a syringosubarachnoid shunt prior to the deformity correction (Fig. 7). An experienced intraoperative monitoring team is sine qua non for the higher risk category.

In patients who have been operated on for tethered cord and demonstrate progressive neurological deficit and significant deformity, it is important to remember that the cord may become retethered unless that diagnosis can be ruled out by the temporal sequence of events and pathology (e.g., detethering within the last few months and low-risk pathologies like lipomatous filum). In one of our patients, who was operated on for myelomeningocele, the cord was stretched over the deformity at L-3 and a simultaneous correction was necessary, as the apex of the progressive deformity was the point of neurological insult and the deformity and retethering both could have been contributory (Fig. 5).

Although we have shown that it is possible to perform concurrent spinal deformity surgery and Chiari decompression and/or untethering procedures safely, it is not always necessary to perform these procedures concurrently.
The decision making has multiple dimensions; sometimes the decision has to be concurrent, sometimes the etiology has to be primarily tackled with staged management, and sometimes the sequence and management are controversial. For instance, in both of our patients with previously treated myelomeningocele, the neurological deficit was most likely secondary to the retethering but aggravated by the deformity, and concurrent surgery was essential (Figs. $3-5$ ). Occasionally, the presence of syringomyelia requires that the etiology be directly addressed first. In other situations (e.g., isolated CM without syringomyelia), staging may be considered optional, although the issue may be controversial. ${ }^{16}$

\section{Nuances and Limitations}

In cases of patients who have undergone myelomeningocele repair in infancy and have previously been operated on for tethered cord, it may be safer to consider prophylactic detethering based on radiology, pathology, and the time gap from the detethering, as previously discussed. Spinal implants are placed before the neurosurgical procedure whenever instability is expected (as with corpectomy and longitudinal implants [rods]) and acute deformity correction is not performed until the neurosurgical procedure is completed (Fig. 2). Neuromonitoring is a key adjunct, particularly when operating on a compromised cord. We prefer aggressive management of syringomyelia via placement of a syringosubarachnoid shunt in case of significant syrinx and holocord syrinx (Figs. 6 and 7). Double tethering has to be ruled out especially in patients with diastematomyelia, where an additional lipomatous filum terminale is a common association (Fig. 1).

Our work, although unique, has limitations. Our main limitations include the small sample size and the descriptive and retrospective nature of this study. Moreover, our comparison with staged surgery was only from the literature.

\section{Conclusions}

Our results clearly confirm that performing deformity correction during the same surgical session as a definitive 
neurosurgical procedure for neurogenic kyphoscoliosis is feasible and can be done without increasing morbidity. Concurrent surgery may provide economic advantages and avoid the additional risk of complications associated with a second surgery.

Our risk stratification of patients with neurogenic kyphoscoliosis requiring a neurosurgical procedure in addition to deformity correction can serve as a guideline to optimize results.

The use of intraoperative electrophysiological monitoring is a key adjunct for avoiding morbidity, especially when the spinal cord is compromised.

\section{References}

1. Ayvaz M, Alanay A, Yazici M, Acaroglu E, Akalan N, Aksoy C: Safety and efficacy of posterior instrumentation for patients with congenital scoliosis and spinal dysraphism. J Pediatr Orthop 27:380-386, 2007

2. Bowman RM, Mohan A, Ito J, Seibly JM, McLone DG: Tethered cord release: a long-term study in 114 patients. J Neurosurg Pediatr 3:181-187, 2009

3. Cardoso M, Keating RF: Neurosurgical management of spinal dysraphism and neurogenic scoliosis. Spine (Phila Pa 1976) 34:1775-1782, 2009

4. Entwistle MA, Patel D: Scoliosis surgery in children. Contin Educ Anaesth Crit Care Pain 6:13-16, 2006

5. Gibson PRJ: Anaesthesia for correction of scoliosis in children. Anaesth Intensive Care 32:548-559, 2004

6. Golumbeanu M, Pop A: Study on child and adolescent idiopathic scoliosis. Arad Med J 13:59-67, 2010

7. Hamzaoglu A, Ozturk C, Tezer M, Aydogan M, Sarier M, Talu U: Simultaneous surgical treatment in congenital scoliosis and/or kyphosis associated with intraspinal abnormalities. Spine (Phila Pa 1976) 32:2880-2884, 2007

8. Mehta VA, Gottfried ON, McGirt MJ, Gokaslan ZL, Ahn ES, Jallo GI: Safety and efficacy of concurrent pediatric spinal cord untethering and deformity correction. J Spinal Disord Tech 24:401-405, 2011

9. Mummareddy N, Dewan MC, Mercier MR, Naftel RP, Wellons JC III, Bonfield CM: Scoliosis in myelomeningocele: epidemiology, management, and functional outcome. J Neurosurg Pediatr 20:99-108, 2017

10. Murans G, Gustavsson B, Saraste H: One-stage major spine deformity correction surgery: comparison between groups with and without additional neurosurgical intervention, with more than 24 months of follow-up. Clinical article. J Neurosurg Spine 13:666-671, 2010
11. Queruz JCF, Kato A, Abreu de Aguiar C, Avila LM, Munhoz da Rocha LE: Evaluation of idiopathic scoliosis by anterior and posterior arthrodesis. Coluna/Columna 14:88-92, 2015

12. Reigel DH, Tchernoukha K, Bazmi B, Kortyna R, Rotenstein D: Change in spinal curvature following release of tethered spinal cord associated with spina bifida. Pediatr Neurosurg 20:30-42, 1994

13. Samdani AF, Asghar J, Pahys J, D’Andrea L, Betz RR: Concurrent spinal cord untethering and scoliosis correction: case report. Spine (Phila Pa 1976) 32:E832-E836, 2007

14. Samdani AF, Fine AL, Sagoo SS, Shah SC, Cahill PJ, Clements DH, et al: A patient with myelomeningocele: is untethering necessary prior to scoliosis correction? Neurosurg Focus 29(1):E8, 2010

15. Shen J, Zhang J, Feng F, Wang Y, Qiu G, Li Z: Corrective surgery for congenital scoliosis associated with split cord malformation: it may be safe to leave diastematomyelia untreated in patients with intact or stable neurological status. J Bone Joint Surg Am 98:926-936, 2016

16. Wang Y, Xie J, Zhao Z, Zhang Y, Li T, Si Y: Changes in CSF flow after one-stage posterior vertebral column resection in scoliosis patients with syringomyelia and Chiari malformation type I. J Neurosurg Spine 18:456-464, 2013

\section{Disclosures}

The authors report no conflict of interest concerning the materials or methods used in this study or the findings specified in this paper.

\section{Author Contributions}

Conception and design: Udayakumaran, Tahasildar. Acquisition of data: Udayakumaran, Tahasildar. Analysis and interpretation of data: Udayakumaran, Tahasildar. Drafting the article: Udayakumaran, Onyia. Critically revising the article: Udayakumaran, Menon. Reviewed submitted version of manuscript: Udayakumaran, Onyia. Approved the final version of the manuscript on behalf of all authors: Udayakumaran. Administrative/technical/ material support: Udayakumaran. Study supervision: Udayakumaran, Menon.

\section{Correspondence}

Suhas Udayakumaran, Division of Paediatric Neurosurgery, Department of Neurosurgery, Amrita Institute of Medical Sciences and Research Centre, Kochi, Kerala 682 041, India. email: dr.suhas@gmail.com. 